\title{
Serum HE4 is more suitable as a biomarker than CA125 in Chinese women with benign gynecologic disorders
}

\author{
Zhang Yan $^{1 *}$, Qiao Chunxia ${ }^{2 *}$, Li Lian ${ }^{3}$, Zhao Xuye $^{4}, \mathrm{Li} \mathrm{Yali}^{1}$
}

1. Department of Gynecology and Obstetrics, the Chinese PLA General Hospital

2. Laboratory of Immunology, Institute of Basic Medical Sciences

3. Department of Microbiology, College of Medical Sciences, Inner Mongolia Medical University

4. Department of Gynecology and Obstetrics, Shanxi Tumor Hospital

*Yan Zhang and Chunxia Qiao contributed equally to this work.

\begin{abstract}
Objective: This study measured the human epididymis protein 4 (HE4) and CA125 levels in Chinese women with benign gynecological disorders.

Material and methods: Sera were obtained from Chinese women prior to surgery for a pelvic mass and HE4 and CA125 levels were determined. The proportions of patients with HE4 and CA125 levels were compared.

Results: There were 68 Chinese women with benign diseases. HE4 levels were less elevated than CA125 (1\% V.S. 29\%, $\mathrm{P}<0.001)$. The significant difference was observed in patients with endometriosis/endometriomas in which HE4 was not elevated patients and CA125 was elevated in 53\% (P<0.001). Serum HE4 level was not elevated in patients with cystadenoma ( $0 \%$ V.S. $23 \%, \mathrm{P}<0.001)$ and in patients with germ cell tumors $(0 \%$ V.S. $5 \%, \mathrm{P}<0.001)$.
\end{abstract}

Conclusion: HE4 was less elevated and more suitable as a biomarker than CA125 in chinese women with benign disease.

Key words: benign gynecological disease, biomarker, CA125, HE4

DOI: http://dx.doi.org/10.4314/ahs.v14i4.20

\section{Introduction}

Ovarian cancer was a leading cause of gynecological cancer-related death in women, but most ovarian cancers were unfortunately diagnosed at an advanced stage when the survival rate was $<20 \%$ despite aggressive surgery and chemotherapy. A significant factor contributing to the high mortality of ovarian cancer was the relatively a symptomatic progression of this disease through the early stages ${ }^{1-3}$. Because cures were rare at advanced clinical stages, it was very important for placing emphasis on early detection to reduce ovarian cancer mortality. The most frequently utilized screening modalities include pelvic examina-

\section{Corresponding author: \\ Li Yali \\ Department of Gynecology and Obstetrics, \\ The Chinese PLA General Hospital, \\ Fuxing Road \#28, Beijing, 100853, China. \\ Tel: 86-10-66875547 \\ Fax: 86-10-66938147 \\ Email: Liyalimed@163.com}

tions, transvaginal ultrasounds, and cancer antigen 125 (CA125) serum marker levels ${ }^{4}$.

CA125, a glycoprotein, was detected from epithelial ovarian carcinoma antigen by Bast in 1983. CA125 was a high-molecular-weight mucin that was enzymatically cleaved and shed from the surface of ovarian cancer cells. The gene for CA125 was cloned in 2001 and called MUC16 because of the similarities between its product and the mucin family of proteins. CA125 was a large transmembrane glycoprotein with a carboxyl terminus, which included a cytoplasmic tail, a phosphorylation site for proteolytic cleavage, and the transmembrane domain.

The biological functions of CA125 were complex but seem to enhance the malignant potential of ovarian cancer cells. CA125 played an important role in cellular adhesion, invasion, and intraperitoneal metastasis. Many studies had shown that CA125 exists in the serum of patients with epithelial ovarian cancer. Only a few normal tissues expressed low levels of CA125 including the endometrium, fallopian tube epithelium, lung parenchyma, and cornea. Significant levels of CA125 were found in 
some benign ovarian tumors. CA125, which was mainly from patients prior to surgery for pelvic mass.

used for the diagnosis of ovarian cancer, but also for All serum samples were obtained from women prior to the operation excision, the index of curative effect ob- surgery for pelvic mass. Blood samples were collected servation after chemotherapy. Although CA125 was and centrifuged at $800 \mathrm{~g}$ for $10 \mathrm{~min}$. Serum was colthe most widely used biomarker in ovarian cancer, lected and frozen at $-80^{\circ} \mathrm{c}$. HE4 levels were compared the sensitivity and specificity of CA125 were far from with those of CA125 measured in the same samples. ideal as its levels were elevated in approximately $80 \%$ of Serum HE4 levels were determined using the HE4 ovarian cancers ${ }^{4-8}$. Human epididymis secretory protein EIA assay (Fujirebio Diagnostics, Göteborg, Swe4 (HE4) was first identified in the epithelium of the den), and serum CA125 levels were measured using the distal epididymis using Northern blot analysis and in CanAg CA125 EIA assay (Fujirebio Diagnostics, Götesitu transcript hybridization, which was referred to as borg, Sweden). For CA125, the normal upper limit was WFDC2 because it contained two whey acidic protein $35 \mathrm{U} / \mathrm{mL}$, whereas that for $\mathrm{HE} 4$ was $114 \mathrm{pM}$. A cut off (WAP) domains and a four-disulphide core compris- point that provided the best accuracy in the study was ing eight cysteine residues. HE4 was found to be also determined. We also determined our own ideal cut over expressed in ovarian cancer, whereas its expression off, corresponding to the highest accuracy

in normal tissues was low. It has been used for the early

screening and differential diagnosis of ovarian cancer, Pathology reports were reviewed at the time of as well as for monitoring disease recurrence and progression. Several studies showed that HE4 had better cation of the benign neoplasms. Patients were stratified sensitivity and specificity in the detection of ovarian by benign disease classification. Percentages of elcancer and discrimination between benign and ma- evated biomarker levels were determined. The P vallignant cases compared with CA125 $5^{9-10}$. ues for comparison of the proportion of patients with elevations in HE4 versus CA125 in the various benign

To date, there are no studies that have examined the histopathological classifications were determined.

sensitivity and specificity of HE4 in Chinese women with benign gynecological disorders compared with CA125. In the present study, we compared HE4 levels with those of CA125, demonstrating that HE4 was less elevated than CA125 in Chinese women with benign gynecologic disorders.

\section{Material and method}

From February 2010 to July 2012, 68 patients were included in a prospective study conducted at our institution. All patients were diagnosed with a pelvic mass and were scheduled for surgical intervention. All patients underwent imaging by pelvic ultrasound. Clinical information was retrieved from the patients' hospital notes. All patients underwent surgical removal of the ovarian mass. Before the collection of biological samples and surgery, all patients were required to give fully informed consent. The protocol was approved by the Local Ethics Committee. The Local Ethics Committee levels of HE4 and CA125 as well as serums obtained

We assessed the serum HE4 and CA125 levels in 68 chinese women diagnosed benign gynecologic disorders. Histopathological results were categorized into 9 subgroups. The cysts subgroup included germinal epithelial inclusion, corpus luteal and simple cysts. The subgroup of benign other comprised normal ovaries with othe benign gynecological findings such as mesosalpinx cyst. The subgroup of benign undefined indicated tumor cannot define because left and right of ovary suffered from two different tumors. Cystadenoma wa a singe group including both serous and mucinous histology. Hydrosalpinx, pelvic tuberculosis and abscess as well as pelvic inflammatory disease were sorted together. Sex cord stromal tumors gathered nonspecific steroid and Sertoli-Leydig cell tumor. Other subgroups encompassed endometriosis and endometriomas, germ cell tumors (mature teratoma) and fibroid.The serum E4 levels for all the 68 cases divided by benign pathological categories were shown in Figure1.
Fig.1 Scatter plot of serum HE4 levels by histopathological classification $*$ Median and $95 \%$ percentile were shown in figure1.

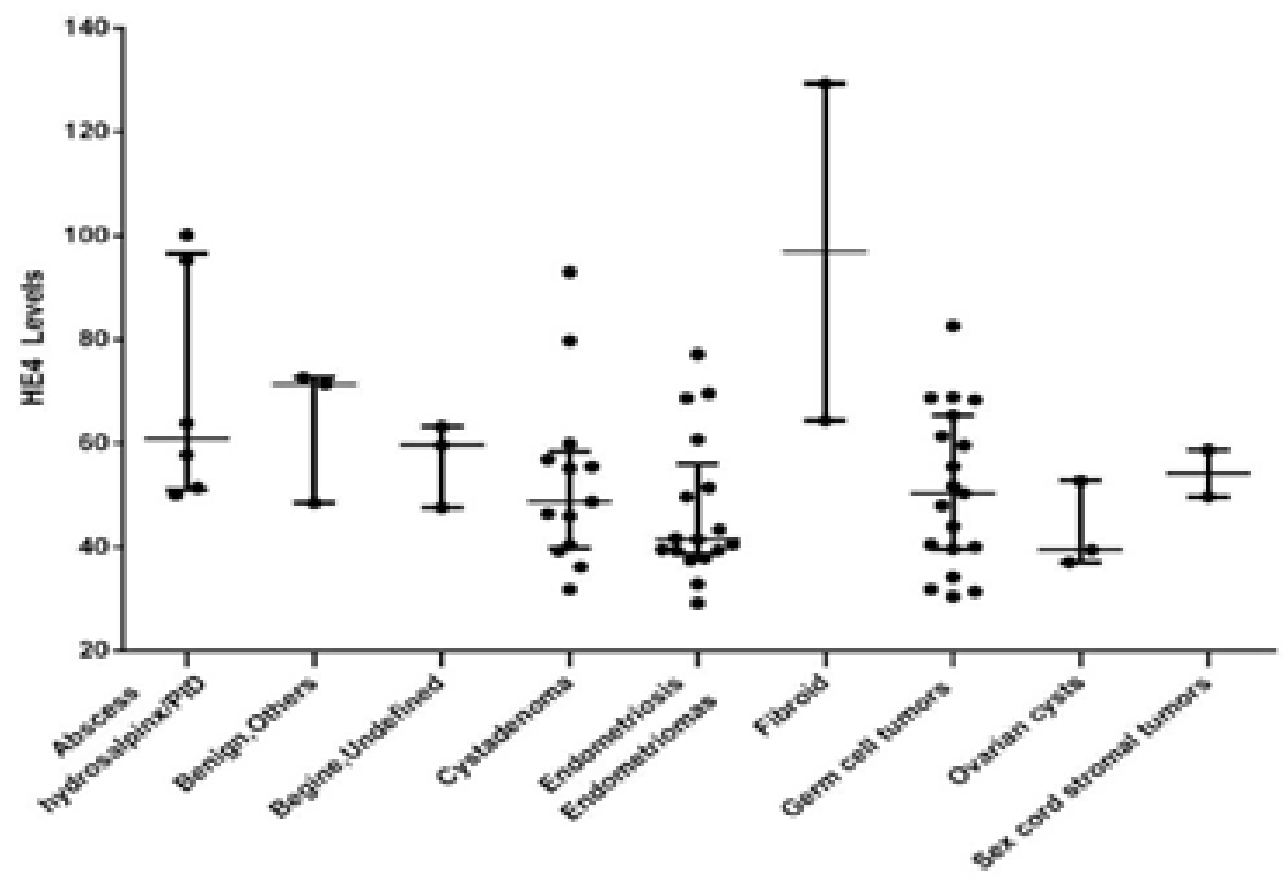

The mean, median, SD and range for HE4 and CA125 grouped by benign hispathological categories were shown in Table1.

Table 1: Analysis of HE4 and CA125 levels by pathological groups

\begin{tabular}{|c|c|c|c|c|c|c|c|}
\hline \multirow[b]{2}{*}{ Benign Classification } & \multirow[b]{2}{*}{$\mathrm{n}$} & \multirow[b]{2}{*}{ Mean } & \multirow{2}{*}{$\frac{\underline{\mathrm{HE} 4(\mathrm{pM})}}{\text { Median }}$} & \multirow[b]{2}{*}{ Range } & \multirow[b]{2}{*}{ Mean } & \multicolumn{2}{|c|}{ CA125 (U/mL) } \\
\hline & & & & & & Median & Range \\
\hline Oraraian crststs & 3 & 43.1 & 39.5 & $37.1-52.8$ & 32.4 & 15.0 & 5.87 .76 .3 \\
\hline $\begin{array}{l}\text { Germ cell tumors (mature } \\
\text { teratoma) }\end{array}$ & 19 & 51.2 & 50.4 & $30.5-82.6$ & 17.9 & 16.6 & $4.5-52.9$ \\
\hline Sex cord stromal tumors & 2 & 54.2 & 54.2 & $49.7-58.7$ & 18.7 & 18.7 & $13.9-23.5$ \\
\hline Cystadenoma & 13 & 53.0 & 48.8 & $31.9-93.0$ & 39.6 & 17.4 & 73.226 .0 \\
\hline Endometriosis/endometriomas & 17 & 47.1 & 41.5 & $29.2-77.2$ & 68.3 & 35.3 & $12.7-200.3$ \\
\hline Benign, Undefined & 3 & 56.8 & 59.6 & $47.6-63.3$ & 20.5 & 21.4 & 17.422 .8 \\
\hline Abscess/hydrosalpinx/PID & 6 & 69.9 & 60.9 & $50.1-100.2$ & 140.5 & 40.6 & 10.4-429.1 \\
\hline Fibroid & 2 & 96.9 & 96.9 & $64.4-129.3$ & 112.2 & 112.2 & 55.9-168.5 \\
\hline Benign, Others(normal ovaries) & 3 & 64.2 & 71.4 & 48.572 .7 & 23.6 & 16.6 & $12.2-41.9$ \\
\hline All benign tumors & 68 & 54.1 & 50.4 & 29.2-129.3 & 49.3 & 22.1 & 4.5429 .1 \\
\hline
\end{tabular}


Comparison of serum HE4 and CA125 levels for all crepancy occurred in endometriosis/endometriothe cases exhibited in Table 2 .

mas subgroup, in which $\mathrm{HE} 4$ was not elevated in 17 Elevation of HE4 and CA125 levels was determined by cases and CA125 was elevated in 53\% (9/17) of the $\mathrm{t}$-test and 95 th percentile cutpoint of $114 \mathrm{pM}$ for HE4 cases $(\mathrm{P}<0.001)$. Astonishingly, serum HE4 levels and $35 \mathrm{U} / \mathrm{mL}$ for CA125 were considered in statisti- in chinese women with cystadenoma were not elcal analysis. In 68 chinese women, elevation of serum evated in 13 cases compared with CA125, which HE4 levels were less than that of CA125. HE4 was was elevated in $23 \%(3 / 13)$ of the cases. Appealingly, found to be elevated in only $1 \%(1 / 68)$ of benign cases compared with CA125 in germ cell tumors, which was compared with serum CA125 levels that were elevated elevated in 1 cases $(1 / 19)$, HE4 levels in serum is les in $29 \%(20 / 68)$ of cases $(\mathrm{P}<0.001)$. Evidence from elevated $(\mathrm{P}<0.001)$. Also, HE4 was significantly less elsubgroup classification of benign gynecological evated compare with CA125 in patients with Ovarian disorders demonstrated that there was a significantly cysts $(0 \%$ V.S. $33 \%, \mathrm{P}=0.002)$. HE4 was equally found difference in the proportion of chinese patients to be less elevated in patients with inflammatory diswhere CA125 was elevated compared with HE4 with ease (abscess, hydrosalpinx, and PID)( $0 \%$ V.S. 50\%, the exception of fibroid. The most significantly dis- $\mathrm{P}=0.046)$. Table 2.

Table 2 :Percentage of elevated biomarker levels in all cases (HE4>114pM and CA125 $>35 \mathrm{U} / \mathrm{mL}$ )

\begin{tabular}{lccc} 
& \multicolumn{3}{c}{ HE4>05th } \\
Benign classification & $\mathrm{n}$ & $\mathrm{n}$ & $\%$ \\
\hline Ovarian cysts & 3 & 0 & 0 \\
Germ cell tumors (mature teratoma) & 19 & 0 & 0 \\
Sex cord stromal tumors & 2 & 0 & 0 \\
Cystadenoma & 13 & 0 & 0 \\
Endometriosis/endometriomas & 17 & 0 & 0 \\
Benign, Undefined & 3 & 0 & 0 \\
Abscess/hydrosalpinx/PID & 6 & 0 & 0 \\
Fibroid & 2 & 1 & 50 \\
Benign, Others(normal ovaries) & 3 & 0 & 0 \\
All benign tumors & 68 & 1 & 1
\end{tabular}

\begin{tabular}{cccc}
$\mathrm{n}$ & $\begin{array}{c}\text { CA125 }>95^{\text {th }} \\
\text { percentile }\end{array}$ & P value \\
\hline 1 & 33 & & 0.002 \\
1 & & 5 & 0.000 \\
0 & & 0 & 0.020 \\
3 & 23 & & 0.000 \\
9 & 53 & & 0.000 \\
0 & & 0 & 0.008 \\
3 & 50 & & 0.046 \\
2 & 100 & & 0.437 \\
1 & 33 & & 0.042 \\
20 & 29 & & 0.000 \\
\hline
\end{tabular}

\section{Discussion} human epididymis and was expressed in some normal tissues $^{11-12}$. Recently, HE4 was regarded as a new biomarker, highly expressed in the early stage of ovarian cancer. However, serum HE4 levels rarely increased in benign gynecologic conditions, suggesting its complementary role to CA125 $5^{13-15}$. Similar to CA125, HE4 levels were significantly higher in patients with ovarian cancer compared with levels observed in patients with benign disease or healthy controls ${ }^{16}$.

Several studies have suggested the combing a serum HE4 assay with a CA125 assay could warrant the detection of gynecologic diseases ${ }^{17-19}$. HE4 assay could complement the classical CA125 assay to improve the detection of patients with ovarian cancer. Moore et al. reported HE4 levels in women with

els. Our founding provides strong evidence the specifiAfrican Health Sciences Vol 14 Issue 4, December 2014 city of HE4 over CA125 and the most useful marker for diagnosis of endometriosis from benign and malignant ovarian mass.

Inflammatory situations such as pelvic inflammatory disease were known to induce high concentrations of CA125 ${ }^{22}$. A raised CA125 levels combined with the presence of pelvic mass suggested an ovarian malignancy ${ }^{23}$. We discovered CA125 was elevated in 50\% patients, which is consistent those reported by Dong et $\mathrm{al}^{24}$, While few patients showed the elevation of HE4.

Several studies have shown CA125 levels were increased in the patients with an ovarian neoplasm ${ }^{25-26}$. In this study, 23\% patients had elevated CA125 levels in contrast to none of patients with elevated levels of HE4. The most common benign neoplasms were serous and mucinous cystadenomas, which accounted for $28.4 \%$ of women with benign disease ${ }^{27}$.

Generally, few patients with benign gynecologic diseases had an elevated HE4 levels compared with that with an elevated CA125 levels ${ }^{27-28}$. This study showed Germ cell tumors (mature teratoma) displayed a smaller fractions of cases with elevated HE4 levels compared with CA125. However, some benign conditions could be associated with high HE4 levels. Recent study had reported high HE4 levels in uterine fibroma ${ }^{30}$.Consistent with this result, in fibroid subgroup, there was no significant difference in the proportion of women, in which both biomarkers were elevated.

Serum HE4, isolated from patients with benign ovaran tumors, had the highest sensitivity as a biomarket, which was $67 \%$, with a specificity of $96 \%{ }^{29}$. The combination of HE4 and CA125 increases the sensitivity of one biomarker alone. In some cases, HE4 had advantages over CA125 in diagnose. An increased of HE4 levels before CA125 increment were strictly associated to a relapse of the disease ${ }^{30}$. In addition, the concentrations of serum HE4 was not influence by the phase of menstrual cycle and hormonal therapy, suggesting a potential role in clinic $^{31-32}$.

By histopathological classification, we collected limited samples of patients with benign ovarian tumor in our study. As a result of this levation of HE4 or CA125 was obvious in some histological types, such as endometriosis/endometriomas, cystadenoma and germ cell tumors, while was not significant in other types, including patients with inflammatory disease (abscess, hydrosalpinx, and PID). Kobel et al. had investigated the expression pattern of 21 different biomarkers

African Health Sciences Vol 14 Issue 4, December 2014 cluding HE4 and CA125 in accordance with hispathological classification and reported that biomarker expression varies between histological types ${ }^{33}$.

Overall, our data confirmed previous report that HE4 levels were not frequent elevated in patients with benign conditions when the proportions of patients with elevated levels of HE4 and CA125 were compared. This result supported HE4 can provide more accurate tool for diagnosis of patients with benign ovarian tumors. Future studies will be performed to evaluate HE4 levels in a larger of samples of sera patients with benign disease in ovarian cancer screening trails. At the same time, healthy controls should be

included as possible to enhance reliability of our results.

Conflict of interest

None of the authors have any conflict of interest.

Reference

1. Earle CC, Schrag D, Neville BA, et al. Effect of surgeon specialty on processes of care and outcomes for ovarian cancer patients. J Natl Cancer Inst. 2006; 98:172-180.

2. Olaitan A, Weeks J, Mocroft A, et al. The surgical management of women with ovarian cancer in the south west of England. Br J Cancer. 2001; 85:1824-1830.

3. Tingulstad S, Skjeldestad FE, Hagen B. The effect of centralization of primary surgery on survival in ovarian cancer patients. Obstet Gynecol. 2003; 102: 499-505. 4. Berek JS, Bast RC Jr. Ovarian cancer screening. The use of serial complementary tumor markers to improve sensitivity and specificity for early detection. Cancer. 1995; 76:2092-2096.

5. Moore RG, Miller MC, Steinhoff MM, et al. Serum HE4 levels are less frequently elevated than CA125 in women with benign gynecologic disorders. Am J Obstet Gynecol 2012; 206:351.e1-8.

6. Bast RC Jr, Klug TL, St John E, et al. A radioimmunoassay using a monoclonal antibody to monitor the course of epithelial ovarian cancer. N Engl J Med 1983; 309:883-887.

7. Buller RE, Berman ML, Bloss JD, Manetta A, Diaia PJ. Serum CA125 regression in epithelial ovarian cancer: correlation with reassessment findings and survival. Gynecol Oncol 1992; 47:87-92

8. Van der Burg ME, Lammes FB, Verweij J. The role of CA 125 in the early diagnosis ofprogressive disease in ovarian cancer. Ann Oncol 1990; 1:301-302.

9. Holcomb K, Vucetic Z, Miller MC, et al. Human epididymis protein 4 offers superior specificity in the differentiation of benign and malignant adnexal mass- 
es in premenopausal women. Am J Obstet Gynecol 2011; 205:358.e1-6.

10. Moore RG, Brown AK, Miller MC, et al. The use of multiple novel tumor biomarkers for the detection of ovarian carcinoma in patients with a pelvic mass. Gynecol Oncol 2008; 108:402-408.

11. Kirchhoff C, Habben I, Ivell R, et al. A major human epididymis-specific cDNA encodes a protein with sequence homology to extracellular proteinase inhibitors[J]. Biology of Reproduction, 1991,45(2): 350-357.

12. Bingle L, Singleton V, Bingle CD. The putative ovarian tumour marker gene HE4 (WFDC2), is expressed in normal tissues and undergoes complex alternative splicing to yield multiple protein isoforms. J]. Oncogene, 2002, 21(17): 2768-2773.

13. Richard GM, Michael CM, Margaret MS, et al. Serum HE4 levels are less frequently elevated than CA125 in women with benign gynecologic disorders[]]. American Journal of Obstetrics and Gynecology, 2012, 206(4): 351. e351-351.e358.

14. Granato T, Midulla C, Longo F, et al. Role of HE4,CA72-4, and CA125 in monitoring ovarian cancer[J]. Tumor Biology, 2012, 33(5): 1335-1339.

15. Yamashita S, Tokuishi K, Hashimoto T, et al. Prognostic significance of HE4 expression in pulmonary adenocarcinoma[J]. Tumor Biology, 2011, 32(2): 265-271.

16. Hamed EO, Ahmed H, Sedeek OB, et al. Significance of HE4 estimation in comparison with CA125 in diagnosis of ovarian cancer and assessment of treatment response[J]. Diagnostic Pathology, 2013, 8(11). doi:10.1186/1746-1596-8-11.

17. Andersen MR, Goff BA, Lowe KA, et al. Use of a Symptom Index, CA125, and HE4 to predict ovarian cancer[J]. Gynecologic Oncology, 2010, 116(3): 378383.

18. Park Y, Lee JH, Hong DJ, et al. Diagnostic performances of HE4 and CA125 for the detection of ovarian cancer from patients with various gynecologic and non-gynecologic diseases[J]. Clinical Biochemistry, 2011, 44(10-11): 884-888.

19. Anastasi E, Granato T, Coppa A, et al. HE4 in the Differential Diagnosis of a Pelvic Mass: A Case Report [J]. International Journal of Molecular Sciences 2011, 12: 627-632.

20. Escudero JM, Auge JM, Filella X, et al. Comparison of Serum Human Epididymis Protein 4 with Cancer Antigen 125 as a Tumor Marker in Patients with Malignant and Nonmalignant Diseases[J]. Clinical Chemistry, 2011, 57(11): 1534-1544.
21. Anastasi E, Granato T, Falzarano R, et al. The use of HE4, CA125 and CA72-4 biomarkers for differential diagnosis between ovarian endometrioma and epithelial ovarian cancer[J]. Journal of Ovarian Research, 2013, 6(44). doi: 10.1186/1757-2215-6-44.

22. Epiney M, Bertossa C, Weil A, et al. CA125 production by the peritoneum: in-vitro and in-vivo studies[]]. Human Reproduction, 2000, 15(6): 1261-1265.

23. Asher V, Hammond R, Duncan TJ. Pelvic mass associated with raised CA 125 for benign condition: a case report[J]. World Journal of Surgical Oncology, 2010, 8(28). doi: 10.1186/1477-7819-8-28.

24. Dong L, Chang XH, Ye X, et al. The values of serum human epididymis secretory protein 4 and CA(125) assay in the diagnosis of ovarian malignancy[J]. Zhonghua Fu Chan Ke Za Zhi, 2008,43(12): 931-936.

25. Yedema C, Massuger L, Hilgers J, et al. Pre-operative discrimination between benign and malignant ovarian tumors using a combination of CA125 and CA15. 3 serum assays[J]. Journal of Cancer, 1988, 41(S3): 61-67. 26. Sedlaczek P, Frydecka I, Gabrys M, et al. Comparative analysis of CA125, tissue polypeptide specific antigen, and soluble interleukin-2 receptor $\alpha$ levels in sera, cyst, and ascitic fluids from patients with ovarian carcinoma[J]. Cancer, 2002, 95(9): 1886-1893.

27. Moore RG, Brown AK, Miller MG, et al. The use of multiple novel tumor biomarkers for the detection of ovarian carcinoma in patients with a pelvic mass[]]. Gynecologic Oncology, 2008,108(2): 402-408.

28. Granato T, Midulla C, Longo F, et al. Role of HE4, CA72.4, and CA125 in monitoring ovarian cancer[J]. Tumor Biology, 2012, 33(5): 1335-1339.

29. HellstromI, Raycraft J, Ledbetter $\mathrm{MH}$, et al. The HE4 (WFDC2) protein is a biomarker for ovarian carcinoma[J]. Cancer Research, 2003, 63(13): 36953700 .

30. Anastasi E, Marchei GG, Viggiani V, et al. HE4: a new potential early biomarker for the recurrence of ovarian cancer[J]. Tumor Biology, 2010, 31(2): 113-119. 31. Anastasi E, Granato T, Marcei GG, et al. Ovarian tumor marker HE4 is differently expressed during the phases of the menstrual cycle in healthy young women[J]. Tumor Biology, 2010, 31(5): 411-415.

32. Hallamaa M, Suvitie P, Huhtinen K, et al. Serum HE4 concentration is not dependent on menstrual cycle or hormonal treatment among endometriosis patients and healthy premenopausal women[J]. Gynecologic Oncology, 2012, 125(3): 667-672.

33. Kobel M, Kalloger SE, Boyd N, et al. Ovarian Carcinoma Subtypes Are Different Diseases: Implications for Biomarker Studies[J]. PLoS Medicine, 2008, 5(12): 1749-1760. 